\title{
Dynamic Pricing with Constant Demand Elasticity
}

\author{
R. Preston McAfee \\ Division of Humanities and Social Sciences \\ California Institute of Technology \\ Pasadena, CA 91125 \\ Phone: (626) 395-3476 \\ Fax: (626) 793-4681 \\ Email: mcafee@hss.caltech.edu, \\ and \\ Vera te Velde \\ Division of Humanities and Social Sciences \\ California Institute of Technology \\ Pasadena, CA 91125 \\ Phone: N/A \\ Fax: N/A \\ Email: vtevelde@gmail.com
}




\title{
Dynamic Pricing with Constant Demand Elasticity
}

\begin{abstract}
The model of Gallego and van Ryzin (1994) is specialized to the case of constant elasticity of demand. A closed form is developed, which has an even simpler form than that arising with exponential demand, and possesses an excellent approximation. It is shown in this environment that monopoly is efficient, which means that all the behavior usually attributed to monopoly pricing is actually a consequence of efficient pricing and would arise even in a perfectly competitive environment. If the initial supply is not too large, it is shown that consumers have no incentive to delay their purchases in order to get a lower price at the average inventory prevailing at any time.
\end{abstract}

Key words: yield management, dynamic pricing, efficient pricing

Submissions and acceptance: Received <month and year>; revision received <month and year>; accepted <month and year> 
Standard dynamic pricing models, ably and thoroughly reviewed in Talluri and van Ryzin (2004), either work with general demand forms or with the special case of exponential demand, where the proportion of people valuing the good above price $p$ comes in the form $e^{-b(p-p)}$, for $p$ $\geq p_{0}$. This special case is used only because it is simple to solve; it is not a standard demand form used by economists. While the general case is obviously preferable to any special case, often the general case resists application, and special cases have their virtues. This short paper offers an alternative special case that arises from the most common demand structure used by economists, and which has other useful analytic properties. ${ }^{1}$ It is shown in particular that with constant elasticity of demand, the monopoly solution is efficient, so that the behavior attributed to monopoly pricing is actually a consequence of efficient pricing, a fact difficult to see in the general model. In addition, it is demonstrated that, if the initial demand is sufficiently large relative to the number of units available, search will be on average unprofitable, supporting the commonly maintained hypothesis that customers either buy immediately or go away.

Consider the (static) demand held by a particular potential customer. Demand functions generally give the quantity $q(p)$ demanded as a function of price $p$. A demand function is said to have constant elasticity if $\varepsilon=-\frac{p q^{\prime}(p)}{q(p)}$ is a constant, in which case we can write $q(p)=a p^{-\varepsilon}$.

Constant elasticity is useful in the dynamic pricing context because it starkly simplifies the monopoly pricing problem. Consider a monopolist facing constant elasticity of demand $\varepsilon$, and with a marginal $\operatorname{cost} c$. This firm's profits are

$$
\pi=\max _{p}(p-c) q(p)=(p-c) a p^{-\varepsilon}
$$

It is straightforward to verify that, first, if $\varepsilon<1$, or if $\varepsilon=1$ and $c>0$, then the problem has no solution. ${ }^{2}$ Second, if $\varepsilon>1$ and $c>0$, the solution to the monopoly pricing problem is

$$
p^{*}=\frac{\varepsilon}{\varepsilon-1} c
$$

It is this fact, that the monopoly price is a constant proportion of marginal cost, which we can exploit to produce a solution to the dynamic pricing problem. The static profits turn out to be

$$
\pi^{*}=a \frac{(\varepsilon-1)^{\varepsilon-1}}{\varepsilon^{\varepsilon}} c^{1-\varepsilon}
$$

From this point on, we assume $\varepsilon>1$. We start with the standard dynamic pricing problem adapted from Gallego and van Ryzin (1994). Consider a good that is valueless if not sold by time $T$. Let

\footnotetext{
${ }^{1}$ Monahan, Petruzzi and Zhao (2004) also considered constant elasticity of demand in a dynamic pricing problem; the connection between that deep paper and the present study after the results are presented. Recent contributions in the theory include Queen et al. (2007), Shen and Su (2007) and Xia and Dube (2007).

${ }^{2}$ The "solution" is to sell an infinitesimal quantity at an infinite price, a fact made evident by considering a discrete version of the problem.
} 
$\lambda(p, t)$ be the Poisson arrival rate of customers willing to pay $p$ at time $t$. Suppose the cost of service is zero. ${ }^{3}$ Let $v_{n}(t)$ represent the continuation value (net of any past sales) to the seller of having $n$ items to sell at time $t$. By hypothesis,

$$
v_{n}(T)=0 .
$$

In addition, having nothing to sell should also be valueless, so that at all $t$,

$$
v_{0}(t)=0 \text {. }
$$

Following Gallego and van Ryzin, the Bellman equation entails

$$
v_{n}^{\prime}(t)=-\max _{p} \lambda(p, t)\left(p-\left(v_{n}(t)-v_{n-1}(t)\right)\right)
$$

Now posit constant elasticity, so that $\lambda(p, t)=a(t) p^{-\varepsilon}$. Then the maximization problem embodied in (6) comes in the same form as before and hence has the solution (1)-(2), which yields

$$
v_{n}^{\prime}(t)=-a(t) \frac{(\varepsilon-1)^{\varepsilon-1}}{\varepsilon^{\varepsilon}}\left(v_{n}(t)-v_{n-1}(t)\right)^{1-\varepsilon}
$$

Define a sequence $\beta_{n}$ by $\beta_{0}=0$ and $\beta_{n}$ as the solution exceeding $\beta_{n-1}$ to $\beta_{n}=\left(\frac{\varepsilon-1}{\varepsilon}\right)^{\varepsilon-1}\left(\beta_{n}-\beta_{n-1}\right)^{1-\varepsilon}$. The values of $\beta_{n}$ exist and are uniquely determined, a fact evident from writing

$$
\beta_{n}\left(\beta_{n}-\beta_{n-1}\right)^{\varepsilon-1}=\left(\frac{\varepsilon-1}{\varepsilon}\right)^{\varepsilon-1}
$$

and noting the left hand side is increasing and divergent in $\beta_{n}$, for $\beta_{n}>\beta_{n-1}$. Moreover, $\beta_{n}-\beta_{n-1}$ is a decreasing function of $n$, a fact evident from $\beta_{n}-\beta_{n-1}=\frac{\varepsilon-1}{\varepsilon}\left(\frac{1}{\beta_{n}}\right)^{\frac{1}{\varepsilon-1}}$ and the fact that $\beta_{n}$ is increasing in $n$. Thus, $\beta_{n}$ is a concave function of $n$.

\footnotetext{
${ }^{3}$ In the airline context, the cost of service includes gasoline, meals, complaint resolution and other costs that depend on a seat being occupied, but may also include the lost opportunity to sell a flight on another aircraft to a particular customer. Most of these costs are quite small, but it is troubling to assume that the value of the opportunity to sell the same individual a different seat is zero.
} 
Let $A(t)=\int_{t}^{T} a(s) d s$. The value $A(t)$ is the expected number of future sales at a price equal to one.

Theorem 1: The solution to (4), (5) and (7) is $v_{n}(t)=\beta_{n}(A(t))^{1 / \varepsilon}$. In addition, the price posted satisfies

$$
p_{n}(t)=\beta_{n}^{-1 / \varepsilon-1} A(t)^{1 / \varepsilon}
$$

Proofs are contained in the Appendix. Theorem 1 establishes a closed form for the solution, given that the terms $\beta_{k}$ are readily computable. However, an approximation of the $\beta_{k}$ terms is available that provides better insight to the behavior of the prices and profits and is remarkably exact. This approximation will also permit approximation of the distribution of items remaining at any given time. Let $q_{n}(t)$ represent the probability that exactly $n$ items remain at time $t$.

Theorem 2: $\operatorname{Lim}_{n \rightarrow \infty} \frac{\beta_{n}}{\frac{\varepsilon-1}{\varepsilon}}=1$. For large $n, p_{n}(t) \approx\left(\frac{A(t)}{n}\right)^{1 / \varepsilon}$, and

$q_{n}(t) \approx\left(\begin{array}{l}N \\ n\end{array}\right)\left(\frac{A(t)}{A(\mathrm{o})}\right)^{n}\left(1-\frac{A(t)}{A(\mathrm{o})}\right)^{N-n}$

The approximation $\beta_{n} \approx\left(n-\frac{1.5}{\varepsilon}\right)^{\frac{\varepsilon-1}{\varepsilon}}$, which approximates equally well in the limit, appears to be within $3 \%$ for $n \geq 2$ and $\varepsilon \geq 1.1$.

An immediate consequence of Theorem 2 is that the expected number of items sold by time $T$ is approximately $N\left(1-\frac{A(t)}{A(\mathrm{o})}\right)$ and the number of items sold is approximately linear in the proportion of the expected customer arrivals.

Further insight into the exact distribution of quantity can be obtained from considering the flow of sales. With an inventory of $n$ at time $t$, the flow of sales is given by

$\lambda(p, t)=a(t) p_{n}(t)^{-\varepsilon}=a(t)\left(\beta_{n}^{\frac{-1}{\varepsilon-1}}(A(t))^{1 / \varepsilon}\right)^{-\varepsilon}=\frac{a(t)}{A(t)} \beta_{n}^{\frac{\varepsilon}{\varepsilon-1}}$ 
Let $\tau_{k}$ represent the random variable which is the time of the $k^{\text {th }}$ sale. Immediately prior to the $k^{\text {th }}$ sale, the inventory is $N-k+1$. Given $\tau_{0}=0$ and conditioning on $\tau_{k-1}$,

$$
\begin{aligned}
\operatorname{Prob}\left\{\tau_{k} \leq t \mid \tau_{k-1}\right\}= & \int_{\tau_{k-1}}^{t} e^{-\int_{\tau_{k-1}}^{s} \lambda\left(p_{N-k+1}(r), r\right) d r} \lambda\left(p_{N-k+1}(s), s\right) \\
& =-e^{-\left.\int_{\tau_{k-1}}^{s} \lambda\left(p_{N-k+1}(r), r\right) d r\right|_{s=\tau_{k-1}} ^{t}}=1-\operatorname{Exp}\left(-\int_{\tau_{k-1}}^{t} \lambda\left(p_{N-k+1}(r), r\right) d r\right) \\
& =1-\operatorname{Exp}\left(-\int_{\tau_{k-1}}^{t} \frac{a(r)}{A(r)} \beta_{N-k+1}^{\frac{\varepsilon}{\varepsilon-1}} d r\right)=1-\left(\frac{A(t)}{A\left(\tau_{k-1}\right)}\right)^{\beta_{N-k+1}^{\frac{\varepsilon}{\varepsilon-1}}}
\end{aligned}
$$

Thus, we see that $\left(\frac{A\left(\tau_{k}\right)}{A\left(\tau_{k-1}\right)}\right)^{\beta_{N-k+1}^{\frac{\varepsilon}{\varepsilon-1}}}$ is uniformly distributed on [0,1], which dramatically simplifies the creation of sample paths. Moreover, theorem 2 yields that $\left(\frac{A\left(\tau_{k}\right)}{A\left(\tau_{k-1}\right)}\right)^{N-k+1}$ is approximately uniformly distributed.

The approximation of theorem 2 readily permits computing approximate price paths. For example, suppose $A(t)=(T-t)^{0.8}$, which insures that more of the customers arrive close to the time the flight departs than before. Specifically, in any interval $[t, T]$, on average about a third more customers arrive in the last half of the interval than in the first half of the interval. Figure 1 shows both the expected price conditional on availability and a symmetric $95 \%$ confidence interval (in grey) for prices as well, for the case of 365 days to sell 200 items, and a demand elasticity of 1.5. Two features of the figure stand out. First, prices rise until the last three days, and the rise is fairly dramatic the last two weeks. A price increase the last month is a salient feature of the data on airline pricing (McAfee and te Velde (2006)), although the price drop at the end is not. The price drop is unavoidable in models where the type of consumer doesn't change over time, because the cost of sale must fall toward the end, and prices fall with the costs. It takes a model with changing demand, such as Zhao and Zheng (2000), to account for this regularity. However, as we see in Figure 1, it is possible with constant elasticity to get rather close. 


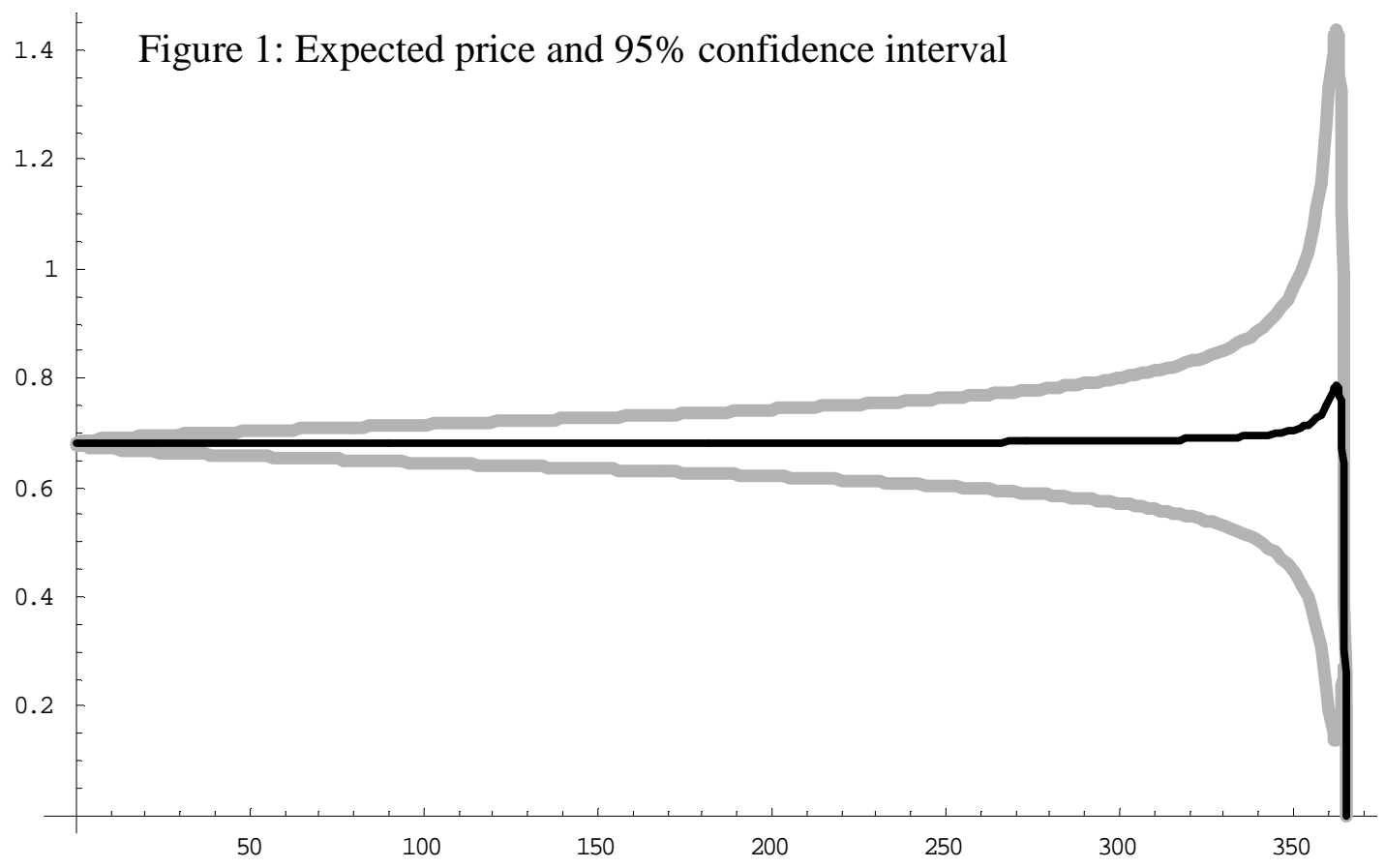

Second, the dispersion of prices grows dramatically toward the end of the period. We believe this to also be a salient feature of real markets, although with less evidence than on average prices. Morever, it is a feature of all Gallego-van Ryzin type models, for the simple reason that the variance of prices is initially zero (there is a deterministic initial price) and is zero at $T$ because the cost of selling any remaining items at the end of the interval is zero.

Among models in this class, the constant elasticity case does not present unreasonable predictions and even does a better job accommodating data than the exponential demand case.

\section{Efficiency}

The prices satisfying equation (9) are prices chosen by a monopoly to maximize profits. What are the socially efficient prices? That is, what would a benevolent dictator choose to maximize the total gains from selling the $N$ items? This question is important because a competitive industry would generally choose prices that maximize social efficiency. Efficiency is a consequence of competition because inefficient offerings are competed away by companies offering higher value to customers along with an increased profit, an action which is possible by reducing the inefficiency. Moreover, examining the efficient solution may provide greater insight than examining an imperfectly competitive industry, such as adeptly introduced by Dana (1994), since it provides a useful benchmark for evaluation for both monopoly and oligopoly.

To identify the socially efficient prices, we first need to identify the value that buyers place on the good. This is subtle because it is mingled with the arrival rate of buyers rather than being a standalone value. To find the expected value, first suppose that we could write the arrival rate $\lambda(x)$ of consumers willing to pay a price $x$ as an arrival rate $\alpha$ times the probability $1-F(x)$ that the consumer is willing to pay the price $x$. (The time dependence is suppressed.) In this case, we have that the expected value of a consumer being willing to pay the price $x$ is 


$$
E\{V \mid V \geq x\}=\int_{x}^{\infty} \frac{v f(v)}{1-F(x)} d v=\int_{x}^{\infty} \frac{\alpha v f(v)}{\alpha(1-F(x))} d v=\int_{x}^{\infty} \frac{-v \lambda^{\prime}(v)}{\lambda(x)} d v
$$

This definition in fact works even in the case where the arrival rate is not necessarily bounded and depends on the posted price. In particular, with our constant elasticity assumption,

$$
E\{V \mid V \geq x\}=\int_{x}^{\infty} \frac{-v \lambda^{\prime}(v)}{\lambda(x)} d v=\int_{x}^{\infty} \frac{\varepsilon v^{-\varepsilon}}{x^{-\varepsilon}} d v=\frac{\varepsilon}{\varepsilon-1} x
$$

Note that the expected value embodied in (10) is larger than the posted price $x$ because any consumer willing to pay $x$ has a value as large as $x$.

The socially efficient price maximizes the buyer value rather than the selling price as a monopolist might maximize. Let $S_{n}(t)$ represent the social value of having $n$ items to sell at time $t$. This social value will satisfy the same endpoint restrictions (4)-(5) that the monopoly value satisfied, and for the same reasons. Then the Bellman equation yields

$$
\begin{aligned}
S_{n}^{\prime}(t) & =-\max _{p} a(t) p^{-\varepsilon}\left(\frac{\varepsilon}{\varepsilon-1} p-\left(S_{n}(t)-S_{n-1}(t)\right)\right) \\
& =-\frac{\varepsilon}{\varepsilon-1} \max _{p} a(t) p^{-\varepsilon}\left(p-\frac{\varepsilon-1}{\varepsilon}\left(S_{n}(t)-S_{n-1}(t)\right)\right)
\end{aligned}
$$

Thus the socially efficient price $p^{\mathrm{e}}$ is the price that maximizes $p^{-\varepsilon}\left(p-\frac{\varepsilon-1}{\varepsilon}\left(S_{n}(t)-S_{n-1}(t)\right)\right)$, and from (1)-(2), that price satisfies

$$
p_{n}^{e}=\frac{\varepsilon}{\varepsilon-1} \frac{\varepsilon-1}{\varepsilon}\left(S_{n}(t)-S_{n-1}(t)\right)=S_{n}(t)-S_{n-1}(t)
$$

Equation (11) is intuitive - the efficient price with $n$ items is just equal to the cost of sale, which is the social value of having $n$ minus the social value of having $n-1$ items to sell. This calculation simplifies the differential equation determining the social value to

$$
S_{n}^{\prime}(t)=-\frac{a(t)}{\varepsilon-1}\left(S_{n}(t)-S_{n-1}(t)\right)^{1-\varepsilon}
$$

Using a development entirely parallel to the monopoly case, it is straightforward to show that (12), along with the endpoint restrictions, imply that there are constants $\gamma_{n}$ so that 


$$
S_{n}(t)=\gamma_{n} A(t)^{1 / \varepsilon}
$$

Moreover, $\gamma_{n}$ satisfies $\gamma_{n}=\frac{\varepsilon}{\varepsilon-1}\left(\gamma_{n}-\gamma_{n-1}\right)^{1-\varepsilon}$ and thus it is readily verified by (8) that

$$
\gamma_{n}=\frac{\varepsilon}{\varepsilon-1} \beta_{n}
$$

From (13)-(14), the efficient price, as a consequence, is

$$
p_{n}^{e}(t)=S_{n}(t)-S_{n-1}(t)=\left(\gamma_{n}-\gamma_{n-1}\right) A(t)^{1 / \varepsilon}=\frac{\varepsilon}{\varepsilon-1}\left(\beta_{n}-\beta_{n-1}\right) A(t)^{1 / \varepsilon}=p_{n}(t)
$$

This construction proves the rather remarkable theorem:

Theorem 3: With constant elasticity of demand, the monopoly prices are socially efficient.

At first glance, theorem 3 seems quite surprising. Generally monopolies wish to reduce sales in order to increase the price at which the good is sold, thereby both increasing revenue and reducing costs. In this case, however, a monopoly prices in exactly the same way that an efficient, or competitive, supplier would price. That is, a competitive industry could not improve on the monopoly solution. This result is a consequence of the nature of marginal revenue with constant elasticity of demand. Efficiency dictates equating the expected prices charged across time - selling the total supply at the same expected price, so that a high value customer is not turned away when a low value customer obtains the good. This is similar to the monopolist's goal, where the monopolist equates marginal revenue - the revenue added by an extra unit of sales - across time. Constant elasticity of demand has the implication that prices and marginal revenues are proportional to each other, so equating one equates the other. The only difference between competition and monopoly, then, would be in total sales, but we found that the solution to the monopoly problem entails selling all the units, so total sales are the same as well. The efficiency of dynamic pricing is discussed in Stokey (1979).

This fact - that with constant elasticity and a fixed supply, monopoly and perfect competition coincide - was already known in another context, the problem of resource extraction. The general economic model is that there is a resource - oil, copper, and iron - with a fixed supply that must be allocated over time. Stiglitz (1976) demonstrates that with constant elasticity, the monopoly price and the competitive price coincide. He also characterizes the effects of increasing elasticity, with a monopoly offering higher prices initially but lower prices subsequently, and thus conserving the resource relative to a competitive market. Stiglitz's contribution involved an infinitely-lived world with a known sequence of demands, and thus is logically distinct from the present analysis, but in light of Stiglitz's discovery, the result that monopoly is efficient is less shocking.

Theorem 3 underscores a more general point. Most of the literature on dynamic pricing has focused, not unreasonably, on the monopoly problem, and attributed the behavior found to 
revenue extraction. In an earlier paper (McAfee and te Velde (2006)), we argued that much of the behavior attributed to monopoly pricing is also a feature of competitive pricing and efficiency. However, the argument here is even more compelling: with constant elasticity of demand, monopoly pricing coincides with competitive pricing, and is efficient.

\section{Search and Arbitrage}

An unrealistic feature of dynamic pricing models is the assumption that consumers do not search. Search in this context could have two distinct effects. First, a consumer willing to pay the current price might wait for a better price. Second, a consumer unwilling to pay the price might return and check for a subsequent better price. It is the first kind that we consider here, and ask whether the pricing structure is such that it pays to wait for a better price, that is, whether consumers can arbitrage the pricing strategy induced by profit maximization.

An immediate simplification is that if it pays to wait, at some point it pays to wait one moment. For the customer, search entails a risk that the good is unavailable. However, if there are at least two units for sale, and the customer waits a negligible amount of time, the risk of not being able to buy is arbitrarily small. Thus, it does not pay to wait if, for all $t$ and $n \geq 2$, and for small delay $\Delta$,

$$
p_{n}(t) \leq a(t) \Delta p_{n-1}(t+\Delta)+(1-a(t) \Delta) p_{n}(t+\Delta)
$$

Inequality (15) states that the price today does not exceed the expected price tomorrow, where the expected price is the price with one fewer item in the inventory in the event that an item sells (with probability $a(t) \Delta$ ), or the reduced price that arises when no sale is made prior to $t+\Delta$. Collecting terms and dividing by $\Delta$, then sending $\Delta$ to zero yields

$$
-p_{n}{ }^{\prime}(t) \leq a(t)\left(p_{n-1}(t)-p_{n}(t)\right)
$$

Using the approximation of theorem 2, inequality (16) reduces to

$$
\frac{1}{\varepsilon} A(t)^{\frac{1}{\varepsilon}-1} a(t) n^{\frac{-1}{\varepsilon}} \leq a(t) A(t)^{\frac{1}{\varepsilon}}\left((n-1)^{\frac{-1}{\varepsilon}}-n^{\frac{-1}{\varepsilon}}\right) \text {, which is equivalent to }
$$

$$
\frac{n-1}{n}\left(1+\frac{1}{\varepsilon A(t)}\right)^{\varepsilon} \leq 1
$$

Generally, there will be some values of $n$ and $t$ that cause (17) to fail, which means it won't be universally true that search is undesirable. If $n$ is large, and $A(t)$ is small, so that there are a lot of remaining items to sell and not much time to sell them, then a delayed purchase will be profitable for the customer. However, at the average level of $n$, search can be undesirable, as the following theorem shows. 
Theorem 4: If $N \leq A(0)$, then a short delay is unprofitable at the average level of capacity prevailing at any given time.

The proof is unappealingly mechanical and relegated to the appendix.

It is possible to provide a somewhat weaker, albeit more complicated, sufficient condition for an immediate purchase to be optimal at the prices prevailing for the expected capacity. Such a weaker condition is not a major improvement on the existing theorem because there will always be circumstances where the conclusion of the theorem fails. If $A(0)$ is small enough relative to $N$, it will pay for a consumer to wait for likely lower future prices. In such instances, the characterization of this paper, and all others using the standard framework, will be inappropriate because customers expected to purchase immediately find it in their interest to delay. On the other hand, we have also demonstrated the positive result that, at the average prevailing capacity, if there are initially more customers than items, a short wait is unprofitable.

Theorem 4 helps justify a heuristic for customer behavior. A customer that checks for a price often won't know how much capacity remains, and thus would find it challenging to determine whether to just buy an item or wait. In such a circumstance, a customer might adopt a heuristic, and do the same thing in all circumstances. Theorem 4 suggests that no simple heuristic, e.g. "wait until a month prior to expiration," is going to dominate just buying at the time the customer arrives.

\section{Conclusions}

This paper examines the standard model of dynamic pricing developed by Gallego and van Ryzin (1994) and establishes a closed form solution for the pricing and value function when demand has constant elasticity. The closed form is substantially simpler than the closed form that arises with exponential demand, primarily because it is separable in the number of remaining items to sell and the expected number of remaining customers. An approximation for the price is developed which is very good for reasonable demand elasticities, and has the appealing form of

$$
\left(\frac{\text { Remaining Customers }}{\text { Remaining Units }}\right)^{1 / \text { elasticity }} \text {. }
$$

An advantage of such a form is that it readily can be taken to data.

The paper then considered the efficient solution and proves the rather counterintuitive proposition that monopoly is efficient from a social perspective. Thus, the usual monopoly distortion - selling too few units - is not present here and all of the behavior usually attributed to monopoly pricing is actually a consequence of efficient allocation. Efficient allocation requires high initial prices to preserve the option of filling later demand, should it arise, and consequently prices are lower toward the expiration of the good.

One potential difference between behavior of a monopolist and efficient behavior involves the number of items made available; a monopolist will generally reduce the quantity in order to 
increase the price charged. In economic language, efficiency requires expected price equal to marginal cost, while a monopolist chooses a quantity equating marginal cost and marginal revenue. The same conclusion applies in the present model; a monopoly would invest in a smaller initial capacity than is efficient. ${ }^{4}$ With constant elasticity of demand, changes in initial inventory adjust the prices by a constant scalar. However, the general conclusion that the pattern of dynamic pricing is a consequence of efficiency rather than monopoly obtains; rejecting efficiency requires actually knowing the marginal cost rather than looking at the price pattern.

Finally, the choice of customers to delay their purchases, hoping to obtain a better price, is considered. It was shown first that if there is a large capacity and few potential remaining customers, customers get a lower price on average by delay. Thus, no general result is going to hold. However, at the prices that prevail for the average level of inventory, it does not pay to delay if capacity is lower than the number of future potential customers. Thus, if customers use a heuristic to determine when to purchase, buying immediately is not a bad choice since more often than not, this is the right thing to do. Improving on immediate purchase is going to require a more clever scheme than, say, "wait until five weeks before the flight."

Other authors have noted that, with constant demand elasticity, inventory enters the optimization in a multiplicatively separable way. Closest to the current analysis is Monahan, Petruzzi and Zhao (2004), which considers a discrete time dynamic pricing problem with random demands. In the current framework, their model would arise if prices were forced to persist for some fixed and non-negligible period of time and could not be dynamically and instantaneously adjusted based on sales during that time. For example, the price might be adjusted only at times $0,1,2$, etc. They then allow for a random scaling factor in the period: where the present study posits that $A(t)-A(t+1)$ is nonstochastic, Monahan, Petruzzi and Zhao permit this value to be a random variable whose distribution is known in advance. They find the same general form of the value function, multiplicative in inventory with the power of one over the elasticity. In addition, they characterize how the shape of the distribution of demand-scaling factors influences the evolution of the prices and value functions; these results have no analog in the present study because $A$ is not random. In contrast, they do not consider the social efficiency of the pricing structure nor the incentives for consumer search. ${ }^{5}$

There are many unresolved mysteries concerning airline prices. Perhaps the most difficult thing to understand is the prevalence of code-shared flights with dramatically different prices. A price difference of $20 \%$ is not uncommon, in spite of the fact that the code-shared flights use the same aircraft and thus offer the same level of inconvenience and discomfort. While some advantage accrues to customers based on frequent flyer programs, even these are exchangeable on codeshared flights involving American Airlines and Alaska Air, and yet we observe substantial difference in fares on such flights. Products that are nearly perfect substitutes sell for dramatically different prices, which challenge any theory embodying customer substitution, as, for example, in Borenstein and Rose (1994). While network effects may account for price

\footnotetext{
${ }^{4}$ We thank Jim Dana for reminding us of this caveat.

${ }^{5}$ Monahan, Petruzzi and Zhao (2004) also endogenize the initial capacity. Because capacity enters in a multiplicatively separable way and the marginal cost of capacity plays no other role, endogenizing capacity is straightforward in either model. Notationally, their $b$ is the elasticity of demand and hence their $m$ is $(\varepsilon-1) / \varepsilon$.
} 
differences - one airline offering expensive code-shared flights as a complement to connecting flights - the price disparity underscores the advantage of search for the consumer.

At the present time, there is little information in the public domain concerning how airline prices are determined. Does the price for a particular flight depend on competitor's prices, overall demand between the relevant cities, demand for complementary flights connecting to other cities, historical demand on that day of the year? If so, one would expect substitute flights to have closely correlated prices, but that is not what we found in McAfee and te Velde (2006). At this point, the mechanism determining airline prices is mysterious, and merits continuing investigation, since airlines engage in the most computationally intensive pricing of any industry. The techniques used by airlines are likely to be copied elsewhere as the ability of companies to condition price offers on other considerations grows with larger databases of customer information and enhanced processing power. 


\section{Appendix: Proofs}

Proof of Theorem 1:

By induction: Equation (5) establishes the base of the induction for $n=0$. Note that (4) is satisfied by the construction of $A$. Suppose that the hypothesis is true for all values less than $k$. From (7)

$v_{k+1}{ }^{\prime}(t)=-a(t) \frac{(\varepsilon-1)^{\varepsilon-1}}{\varepsilon^{\varepsilon}}\left(v_{k+1}(t)-v_{k}(t)\right)^{1-\varepsilon}=-a(t) \frac{(\varepsilon-1)^{\varepsilon-1}}{\varepsilon^{\varepsilon}}\left(v_{k+1}(t)-\beta_{k}(A(t))^{1 / \varepsilon}\right)^{1-\varepsilon}$

This is a linear ordinary differential equation, so we need only verify that the solution holds:

$$
\begin{aligned}
v_{k+1}^{\prime}(t) & +a(t) \frac{(\varepsilon-1)^{\varepsilon-1}}{\varepsilon^{\varepsilon}}\left(v_{k+1}(t)-\beta_{k}(A(t))^{1 / \varepsilon}\right)^{1-\varepsilon} \\
& =\beta_{k+1} \frac{1}{\varepsilon}(A(t))^{1 / \varepsilon^{-1}} A^{\prime}(t)+a(t) \frac{(\varepsilon-1)^{\varepsilon-1}}{\varepsilon^{\varepsilon}}\left(\beta_{k+1}(A(t))^{1 / \varepsilon}-\beta_{k}(A(t))^{1 / \varepsilon}\right)^{1-\varepsilon} \\
& =-\beta_{k+1} \frac{1}{\varepsilon}(A(t))^{\frac{1-\varepsilon}{\varepsilon}} a(t)+a(t) \frac{(\varepsilon-1)^{\varepsilon-1}}{\varepsilon^{\varepsilon}}\left(\beta_{k+1}-\beta_{k}\right)^{1-\varepsilon}(A(t))^{\frac{1-\varepsilon}{\varepsilon}} \\
& =(A(t))^{\frac{1-\varepsilon}{\varepsilon}} a(t)\left(-\beta_{k+1} \frac{1}{\varepsilon}+\frac{(\varepsilon-1)^{\varepsilon-1}}{\varepsilon^{\varepsilon}}\left(\beta_{k+1}-\beta_{k}\right)^{1-\varepsilon}\right)=0
\end{aligned}
$$

which establishes the hypothesis at $k+1$ as desired.

Given the formula for $v_{n}$, the price posted satisfies

$$
p_{n}(t)=\frac{\varepsilon}{\varepsilon-1}\left(v_{n}(t)-v_{n-1}(t)\right)=\frac{\varepsilon}{\varepsilon-1} A(t)^{1 / \varepsilon}\left(\beta_{n}-\beta_{n-1}\right)=\beta_{n}^{-1 / \varepsilon-1} A(t)^{1 / \varepsilon}
$$

since $\beta_{n}-\beta_{n-1}=\frac{\varepsilon-1}{\varepsilon} \beta_{n}^{-1 / \varepsilon-1}$.

Q.E.D. 
Proof of Theorem 2:

Define $\gamma_{n}=\frac{\beta_{n}}{n^{\frac{\varepsilon-1}{\varepsilon}}}$. The theorem states that $\gamma_{n}$ converges to 1 . Using (8),

we have

$$
\gamma_{n} n^{\frac{\varepsilon-1}{\varepsilon}}\left(\gamma_{n} n^{\frac{\varepsilon-1}{\varepsilon}}-\gamma_{n-1}(n-1)^{\frac{\varepsilon-1}{\varepsilon}}\right)^{\varepsilon-1}=\left(\frac{\varepsilon-1}{\varepsilon}\right)^{\varepsilon-1}
$$

or

$$
\gamma_{n}^{\frac{1}{\varepsilon-1}} n^{\frac{1}{\varepsilon}}\left(\gamma_{n} n^{\frac{\varepsilon-1}{\varepsilon}}-\gamma_{n-1}(n-1)^{\frac{\varepsilon-1}{\varepsilon}}\right)=\frac{\varepsilon-1}{\varepsilon}
$$

or

$$
\gamma_{n}^{\frac{1}{\varepsilon-1}} n\left(\gamma_{n}-\gamma_{n-1}\left(\frac{n-1}{n}\right)^{\frac{\varepsilon-1}{\varepsilon}}\right)=\frac{\varepsilon-1}{\varepsilon}
$$

Claim 1: $\gamma_{n} \leq 1$.

Proof of Claim 1: Note that $\gamma_{1}=\beta_{1}=\left(\frac{\varepsilon-1}{\varepsilon}\right)^{\frac{\varepsilon-1}{\varepsilon}}<1$. Suppose, by way of contradiction, that $\gamma_{m}$ is the first instance of $\gamma_{m}>1$. Then $\gamma_{m}>1 \geq \gamma_{m-1}$. Thus

$$
\begin{aligned}
& \frac{\varepsilon-1}{\varepsilon}=\gamma_{m}^{\frac{1}{\varepsilon-1}} m\left(\gamma_{m}-\gamma_{m-1}\left(\frac{m-1}{m}\right)^{\frac{\varepsilon-1}{\varepsilon}}\right) \geq \gamma_{m}^{\frac{1}{\varepsilon-1}} m\left(\gamma_{m}-\gamma_{m}\left(\frac{m-1}{m}\right)^{\frac{\varepsilon-1}{\varepsilon}}\right) \\
& =\gamma_{m}^{\frac{\varepsilon}{\varepsilon-1}} m\left(1-\left(\frac{m-1}{m}\right)^{\frac{\varepsilon-1}{\varepsilon}}\right) \geq \gamma_{m}^{\frac{\varepsilon}{\varepsilon-1}} \frac{\varepsilon-1}{\varepsilon},
\end{aligned}
$$


since $m\left(1-\left(\frac{m-1}{m}\right)^{\frac{\varepsilon-1}{\varepsilon}}\right)$ is a decreasing sequence that converges to $\frac{\varepsilon-1}{\varepsilon}$. This verifies claim 1.

Now rewrite

$$
\gamma_{n}^{\frac{1}{\varepsilon-1}} n\left(\gamma_{n}-\gamma_{n-1}\left(\frac{n-1}{n}\right)^{\frac{\varepsilon-1}{\varepsilon}}\right)=\frac{\varepsilon-1}{\varepsilon}
$$

to obtain

$$
\gamma_{n}=\frac{\varepsilon-1}{n \varepsilon} \gamma_{n}^{\frac{-1}{\varepsilon-1}}+\gamma_{n-1}\left(\frac{n-1}{n}\right)^{\frac{\varepsilon-1}{\varepsilon}} \geq \frac{\varepsilon-1}{n \varepsilon}+\gamma_{n-1}\left(\frac{n-1}{n}\right)^{\frac{\varepsilon-1}{\varepsilon}}
$$

with the inequality implied by claim 1.

Equality in this expression defines a new sequence $\eta_{n}$ which is a lower bound for $\gamma_{n}$.

$$
\eta_{n}=\frac{\varepsilon-1}{n \varepsilon}+\eta_{n-1}\left(\frac{n-1}{n}\right)^{\frac{\varepsilon-1}{\varepsilon}}
$$

It is readily verified by induction that

$$
\begin{aligned}
\eta_{n}=\left(\frac{1}{n}\right)^{\frac{\varepsilon-1}{\varepsilon}} \eta_{\mathrm{o}}+\frac{\varepsilon-1}{\varepsilon} \sum_{j=1}^{n} \frac{1}{j}\left(\frac{j}{n}\right)^{\frac{\varepsilon-1}{\varepsilon}} \\
=\left(\frac{1}{n}\right)^{\frac{\varepsilon-1}{\varepsilon}} \eta_{\mathrm{o}}+\frac{\varepsilon-1}{\varepsilon} \frac{1}{n} \sum_{j=1}^{n}\left(\frac{j}{n}\right)^{\frac{-1}{\varepsilon}} \rightarrow \frac{\varepsilon-1}{\varepsilon} \int_{0}^{1} x^{-1 / \frac{\varepsilon}{\varepsilon}} d x=1 .
\end{aligned}
$$

Thus, $\gamma_{n}$ is bounded between $\eta_{n}$ and 1 and thus converges to 1 .

From (9): $p_{n}(t)=\beta_{n}^{\frac{-1}{\varepsilon-1}}(A(t))^{1 / \varepsilon} \approx\left(\frac{A(t)}{n}\right)^{1 / \varepsilon}$. 
The evolution of the probability that there are $n$ items available at time $t$ is governed by the differential equation

$$
\begin{aligned}
q_{n}^{\prime}(t) & =\lambda\left(p_{n+1}(t), t\right) q_{n+1}(t)-\lambda\left(p_{n}(t), t\right) q_{n}(t) \\
& =a(t)\left(p_{n+1}(t)\right)^{-\varepsilon} q_{n+1}(t)-a(t)\left(p_{n}(t)\right)^{-\varepsilon} q_{n}(t) \\
& =a(t)\left(\beta_{n+1}^{\varepsilon / \varepsilon-1} A(t)^{-1} q_{n+1}(t)-\beta_{n}^{\varepsilon / \varepsilon-1} A(t)^{-1} q_{n}(t)\right) \\
& =\frac{a(t)}{A(t)}\left(\beta_{n+1}^{\varepsilon / \varepsilon-1} q_{n+1}(t)-\beta_{n}^{\varepsilon / \varepsilon-1} q_{n}(t)\right)
\end{aligned}
$$

because $q_{n}$ increases when a sale is made starting with $n+1$ items, and is decreased when a sale is made when $n$ items remain. If the firm begins with $N$ units at time 0 , then $q(N, 0)=1$ and $q(n, 0)=0$ for all $n<N$.

Using the approximation, this becomes

$$
q_{n}^{\prime}(t) \approx \frac{a(t)}{A(t)}\left((n+1) q_{n+1}(t)-n q_{n}(t)\right)
$$

which has the elegant binomial solution:

$$
q_{n}(t) \approx\left(\begin{array}{l}
N \\
n
\end{array}\right)\left(\frac{A(t)}{A(\mathrm{o})}\right)^{n}\left(1-\frac{A(t)}{A(\mathrm{o})}\right)^{N-n}
$$

\section{Q.E.D.}

Proof of Theorem 4:

The expected value of the amount of remaining capacity, $n$ is approximately $n \approx \frac{N A(t)}{A(0)}$.

Inequality (17) is equivalent to this holding for all $t$, but it is more convenient to express it in terms of $n$, with $A(t) \approx \frac{n A(0)}{N}$. Then (17) can be expressed as $\frac{n-1}{n}\left(1+\frac{N}{\varepsilon n A(\mathrm{o})}\right)^{\varepsilon} \leq 1$.

Let $\kappa(x)=(1-x)\left(1+\frac{N}{\varepsilon A(0)} x\right)^{\varepsilon} ;$ It is sufficient to prove that $\kappa(1 / n) \leq 1$ for all $n$ in $[1, N]$. 


$$
\begin{aligned}
& \kappa^{\prime}(x)=-\left(1+\frac{N}{\varepsilon A(\mathrm{o})} x\right)^{\varepsilon}+(1-x)\left(1+\frac{N}{\varepsilon A(\mathrm{o})} x\right)^{\varepsilon-1} \frac{N}{A(\mathrm{o})} \\
& =\left(1+\frac{N}{\varepsilon A(\mathrm{o})} x\right)^{\varepsilon-1}\left[-\left(1+\frac{N}{\varepsilon A(\mathrm{o})} x\right)+(1-x) \frac{N}{A(\mathrm{o})}\right] \\
& =\left(1+\frac{N}{\varepsilon A(\mathrm{o})} x\right)^{\varepsilon-1}\left[\frac{N}{A(\mathrm{O})}-1-x \frac{N}{A(\mathrm{o})}\left(1+\frac{1}{\varepsilon}\right)\right] \\
& =\frac{1}{A(\mathrm{o})}\left(1+\frac{N}{\varepsilon A(\mathrm{o})} x\right)^{\varepsilon-1}\left[N-A(\mathrm{O})-N x\left(1+\frac{1}{\varepsilon}\right)\right] \\
& \leq \frac{1}{A(\mathrm{o})}\left(1+\frac{N}{\varepsilon A(\mathrm{o})} x\right)^{\varepsilon-1}\left[N-A(\mathrm{o})-\left(1+\frac{1}{\varepsilon}\right)\right] \leq \mathrm{o} .
\end{aligned}
$$

Thus, $\kappa(1 / n) \leq \kappa(1 / N)=(1-1 / N)\left(1+\frac{1}{\varepsilon A(0)}\right)^{\varepsilon} \leq(1-1 / A(0))\left(1+\frac{1}{\varepsilon A(0)}\right)^{\varepsilon} \leq 1$.

The first inequality follows from $n \leq N$ and the fact that $\kappa$ was shown to be decreasing; the second inequality from the hypothesis of the theorem that $N \leq A(0)$, and the third inequality by noting that $(1-z)\left(1+\frac{z}{\varepsilon}\right)^{\varepsilon}$ is a decreasing function of $z$, and thus maximized at $z=0$, so that $(1-z)\left(1+\frac{z}{\varepsilon}\right)^{\varepsilon} \leq 1$

Q.E.D. 
Acknowledge presentations, support, and assistance here 


\section{References}

Borenstein, S., N. Rose. 1994. Competition and price dispersion in the U.S. airline industry. Journal of Political Economy 103, 653-683.

Dana, Jr., J. D. 1999. Equilibrium price dispersion under demand uncertainty: The roles of costly capacity and market structure. RAND Journal of Economics 30(4) 632-660.

Gallego, G., G. van Ryzin. 1994. Optimal dynamic pricing of inventories with stochastic demand over finite horizons. Management Science 40(8) 999-1020.

McAfee, R. P., V. te Velde. 2007. Dynamic pricing in the airline industry. T. J. Hendershott, ed. Handbook on Economics and Information Systems. Elsevier.

Monahan, G. E., N. C. Petruzzi, W. Zhao. 2004. The dynamic pricing problem from a newsvendor's perspective. Manufacturing and Service Operations Management 6(1) 73-91.

Queen, C. C., M. Ferguson, J. Higbie, R. Kapoor. 2007. A comparison of unconstraining methods to improve revenue management systems. Production and Operations Management 16(6) 729-746.

Shen, Z. M.., X. Su. 2007. Customer behavior modeling in revenue management and auctions: A review and new research opportunities. Production and Operations Management 16(6) 713-728.

Stiglitz, J. E. 1976. Monopoly and the rate of extraction of exhaustible resources. The American Economic Review 66(4) 655-661.

Stokey, N. L. 1979. Intertemporal price discrimination. The Quarterly Journal of Economics 93(3) 355-371.

Talluri, K. T., G. J. van Ryzin. 2004. The theory and practice of revenue management. Kluwer Academic Publishers, The Netherlands.

Xia, C. H., P. Dube. 2007. Dynamic pricing in e-services under demand uncertainty. Production and Operations Management 16(6) 701-712.

Zhao, W., Y. Zheng. 2000. Optimal dynamic pricing for perishable assets with nonhomogeneous demand. Management Science 46(3) 375-388. 